\title{
Comparación de índices de abundancia de polaca (Micromesistius australis) a partir de dos fuentes de información mediante la aplicación de modelos lineales mixtos
}

\author{
Francisco Zumpano*, Gonzalo Troccoli, Federico Gorini, Anabela Zavatteri y Emiliano Di Marco \\ Instituto Nacional de Investigación y Desarrollo Pesquero (INIDEP), Paseo Victoria Ocampo No 1, Escollera Norte, B7602HSA - \\ Mar del Plata, Argentina
}

Marine and Fishery Sciences MAFIS

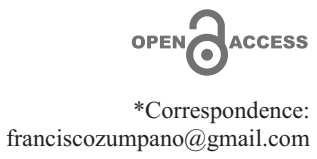

Received: 1 April 2020 Accepted: 18 June 2020

ISSN 2683-7595 (print) ISSN 2683-7951 (online)

https://ojs.inidep.edu.ar

Journal of the Instituto Nacional de Investigación y Desarrollo Pesquero (INIDEP) Creative Commons AttributionNonCommercial-ShareAlike 4.0 International License
RESUMEN. En el presente trabajo se realizó la estandarización de la captura por unidad de esfuerzo (CPUE) de polaca (Micromesistius australis) accesible a la flota surimera que operó en el Océano Atlántico Sudoccidental durante el período 1993-2018. Se aplicaron modelos lineales mixtos (MLM), a partir de la información de la estadística pesquera $\left(\mathrm{MLM}_{\mathrm{EST}}\right)$ y la colectada por los observadores a bordo $\left(\mathrm{MLM}_{\mathrm{OBS}}\right)$ de los buques que componen dicha flota, con el objetivo de comparar los modelos realizados a partir de ambas fuentes de información y el modelo utilizado para la estimación de índices de abundancia de polaca. La aplicación de los MLM mejoró el ajuste con respecto al modelo utilizado hasta la actualidad, sin variar abruptamente la tendencia estimada, lo cual permitiría predecir con mayor exactitud los valores medios de CPUE estandarizados a lo largo de los años. El MLM $\mathrm{OBS}_{\mathrm{BS}}$ mostró un mejor ajuste y una mayor variabilidad explicada que el $\mathrm{MLM}_{\mathrm{EST}}$. A fin de obtener estimaciones más certeras y, de esta forma poder calibrar adecuadamente los modelos de evaluación de la especie, se recomienda utilizar ambas fuentes de información, teniendo en cuenta el mayor o menor grado de representatividad de los datos.

Palabras clave: Biología pesquera, CPUE, estadística pesquera, observadores a bordo.

Comparison of abundance index of Southern blue whiting (Micromesistius australis) from two sources of information by applying mixed linear models

ABSTRACT. In this work, the standardization of the catch per unit effort (CPUE) of the Southern blue whiting (Micromesistius australis) captured by the surimi fleet that operated in the Southwestern Atlantic Ocean during the period 1993-2018 was carried out. Linear mixed models (LMM) were applied to standardize catch and effort data from two different information sources: fishery statistics $\left(\mathrm{LMM}_{\mathrm{EST}}\right)$ and the observers on board of commercial fleet $\left(\mathrm{LMM}_{\mathrm{OBS}}\right)$. These models were compared between them and with that used nowadays to estimate abundance indices. LMM fit better and no significant change was observed in the index tendency, which would allow more accurate prediction of the mean standardized CPUE values over the years. $\mathrm{LMM}_{\mathrm{OBS}}$ showed a better adjust and greater explained variability than the $\mathrm{LMM}_{\mathrm{EST}}$. With the aim of obtaining accurate estimations and, in this way to be able to adequately calibrate the stock assessment model of the species, it is recommended to use both sources of information, taking into account the greater or lesser degree of representativeness of the data.

Key words: Fishery biology, CPUE, fishery statistics, on-board observers. 


\section{INTRODUCCIÓN}

La polaca (Micromesistius australis) es una especie demersal-pelágica que se distribuye en los dos océanos que circundan el cono sur de Sudamérica, además de encontrarse en el Mar de Scotia, alrededor de las islas Georgias, Shetland y Orcadas del Sur (Kock et al. 1985; Cousseau 1993). En el Atlántico Sur, se encuentra entre las latitudes $37^{\circ}$ $\mathrm{S}$ y $56^{\circ} \mathrm{S}$. En cercanías de la latitud $47^{\circ} \mathrm{S}$ su distribución muestra una diferenciación: al norte sólo se la captura en la zona del talud y al sur sus cardúmenes se expanden sobre la plataforma continental. Esta particular presencia está estrechamente relacionada con las aguas de origen subantártico de la Corriente de Malvinas (Perrotta 1982). En este océano, existe un área de reproducción de la especie al suroeste de las Islas Malvinas, donde los individuos de polaca se concentran para tal fin durante los meses de septiembre y octubre (Weiss 1974; Chiechomsky et al. 1981; Wöhler et al. 2004). En los meses estivales se desplazarían hacia el sector oceánico del Mar de Scotia con el objetivo de alimentarse de las elevadas concentraciones de krill disponibles en el área (Wöhler et al. 2001).

En el Pacífico Sudoriental, la especie se distribuye desde los $42^{\circ} \mathrm{S}$ hacia el sur hasta los $57^{\circ} \mathrm{S}$ (Cohen et al. 1990; Lillo et al. 2005; Aguayo et al. 2010). Allí, existe un proceso migratorio estacional que se inicia en el mes de junio cuando ejemplares adultos arriban desde el sur al área de reproducción $\left(47^{\circ} \mathrm{S}-51^{\circ} \mathrm{S}\right)$ en las cercanías del Golfo de Penas, donde se concentran entre agosto y septiembre (Céspedes et al. 1998). En noviembre iniciarían un desplazamiento trófico hacia el sur, abandonando el Océano Pacífico en dirección sureste (Lillo et al. 1999).

Es importante destacar que en el Océano Atlántico una parte importante del stock está presente durante todo el año en un área situada en cercanías de la zona de desove y hasta el sur de la Isla de los Estados, en donde es capturada por las flotas que operan sobre el recurso (Wöhler et al. 2004).

En la Argentina, la polaca representa una especie de gran importancia comercial y es capturada principalmente por los buques surimeros argentinos que operan en su área de distribución (Giussi y Zavatteri 2017). Hacia el final de la década de los setenta, este recurso comenzó a ser explotado intensamente debido a la actividad de buques pesqueros polacos alrededor de las Islas Malvinas, alcanzando un máximo de captura histórico de 258.000 t en 1983 (Wöhler et al. 2001; Gorini y Giussi 2018). A partir de 1993, la pesquería comenzó a ser regulada mediante el establecimiento de una cuota anual de captura (Wöhler et al. 2007) y luego, a partir de 2010, se estableció el sistema de Cuotas Individuales Transferibles de Captura con el objetivo de mejorar la sostenibilidad del recurso. En los años siguientes, el número de buques que pescaba la especie de forma dirigida disminuyó notoriamente, quedando en la actualidad un solo buque operativo. En 2018, la captura declarada en el Atlántico Sudoccidental fue de 11.519 t (Gorini y Giussi 2019).

El manejo del recurso por la administración pesquera argentina surge a partir de recomendaciones del Instituto Nacional de Investigación y Desarrollo Pesquero (INIDEP) basadas en la abundancia y potencial pesquero del stock (Wöhler et al. 2007). Con el objetivo de realizar la estimación de dicha abundancia, se han utilizado modelos de evaluación que requieren de, al menos, un índice de abundancia para su calibración. En la polaca se ha empleado la serie anual de captura por unidad de esfuerzo (CPUE) estandarizada, expresada como toneladas de polaca por hora de pesca (Giussi et al. 2016; Giussi y Zavatteri 2017; Zavatteri y Giussi 2018).

Con el fin de estandarizar la CPUE, se han utilizado desde 1998 hasta la actualidad modelos estadísticos lineales tradicionales o ML (Cordo y Wöhler 1998; Giussi et al. 2013, 2016; Giussi y Zavatteri 2017; Zavatteri y Giussi 2018). Si bien se lograron ajustes aceptables y se cumplieron con los supuestos necesarios para su correcta 
aplicación, este tipo de modelos asumen independencia entre las observaciones que provienen de un mismo buque o de una misma área-tiempo (i.e., rectángulo estadístico-año), sin tener en cuenta la estructura de dependencia que presentan dichos datos. En el primero de los casos, esta dependencia es debida a las diferencias que existen entre los buques en cuanto a sus atributos, capacidad de pesca, tripulación y área de cobertura, y en el segundo, debido a que los rendimientos de un mismo rectángulo estadístico pueden diferir a lo largo de los años y afectar la CPUE. Esto se conoce comúnmente como "pseudoréplica" y afecta directamente a la estimación de la incertidumbre. Los modelos lineales mixtos (MLM) tienen en cuenta este efecto y lo corrigen (Pinheiro y Bates 2000; Crawley 2002; Zuur et al. 2007, 2009). Además, las interacciones comúnmente planteadas en los ML, suelen estar desbalanceadas en la matriz de diseño del modelo, lo que impide el uso de las mismas como variables explicativas. En este sentido, los MLM permiten tener en cuenta el efecto de dichas interacciones como variables aleatorias, aún desbalanceadas, aprovechando la totalidad de la información.

Hasta la actualidad, los datos utilizados para la construcción de la CPUE estandarizada fueron tomados de las capturas declaradas en los partes de pesca (Cordo y Wöhler 1998; Giussi et al. 2013, 2016; Giussi y Zavatteri 2017; Zavatteri y Giussi 2018), provenientes de las bases compiladas por la Secretaría de Agricultura, Ganadería y Pesca (SAGyP) de la República Argentina. Sin embargo, existe otra fuente de información proveniente del "Programa Observadores a Bordo de la Flota Comercial Argentina" del INIDEP que aporta una gran cantidad de información biológico-pesquera detallada y confiable, ya que proporciona datos más certeros sobre capturas y esfuerzo, además de brindar información sobre el bycatch, el descarte y la estructura poblacional de las capturas, entre otras.

En el presente trabajo, se realizó la estandarización de la CPUE de la polaca accesible a la flota surimera que operó durante el período 1993 2018 en el Atlántico Sudoccidental, mediante la aplicación de MLM a partir de la información colectada de la estadística pesquera y por los observadores a bordo de buques comerciales. El objetivo del mismo fue comparar entre sí y con el aplicado en la actualidad (Zavatteri y Giussi 2019), los modelos obtenidos a partir de ambas fuentes de información. Además, se analizó cuál de ellos sería más correcto y factible de ser utilizado en la calibración del modelo de evaluación empleado para la especie.

\section{MATERIALES Y MÉTODOS}

Se utilizó la información de CPUE $\left(\mathrm{kg} \mathrm{h}^{-1}\right) \mathrm{y}$ datos temporales (año y mes) y espaciales (rectángulo estadístico) de las capturas de polaca durante el período 1993-2018, proveniente de dos fuentes de información:

- "Programa Observadores a Bordo de la Flota Comercial Argentina" del INIDEP.

- Estadística oficial derivada de los partes de pesca que son registrados por la SAGyP.

En la primera, tanto los datos de captura como el peso de la misma y las horas de arrastre se encuentran detallados lance a lance, mientras que, en la segunda, dichas variables están informadas por grupos de lances.

Respecto a los datos provenientes de los observadores a bordo, se incluyeron en el análisis sólo aquellos lances de pesca en los cuales la duración del arrastre fue de $20 \mathrm{~min}$ a $15 \mathrm{~h}$, tiempos mínimo y máximo factibles estimados en una operación de pesca (Giussi y Zavatteri 2017; Zavatteri y Giussi 2018). Solo se incluyeron aquellos buques con dos o más años de registros de capturas y más de diez lances por año. Además, se eliminaron del análisis aquellas áreas que contaron con menos de diez registros. 
La estandarización de la CPUE anual a partir de ambas fuentes de información se realizó por medio de la aplicación de MLM. A fines comparativos, en ambos modelos se consideraron los mismos factores fijos y aleatorios. Se utilizó, como variable respuesta, el logaritmo natural de la CPUE y se asumió distribución normal de los errores y de los efectos aleatorios. Dentro de la estructura de la componente fija, se incorporaron factores espacio-temporales considerados importantes y factibles de haber influido sobre la CPUE, tales como el año (AÑO: variable categórica con 26 niveles, 1993 a 2018), el mes (MES: variable categórica con 12 niveles, enero a diciembre) y el rectángulo estadístico (ÁREA: variable categórica con 17 niveles en datos de observadores a bordo y 16 niveles en estadística pesquera). Debido a que los valores de CPUE provenientes de un mismo buque (BUQUE: variable categórica con 6 niveles) y de una misma unidad año-área (AÑO $\times$ ÁREA) estarían correlacionados entre sí, dichos factores se incluyeron dentro de la estructura de la componente aleatoria del modelo.

La estimación de los parámetros de los MLM se realizó mediante parametrización sigma-restrictiva (Hernández 2004). La selección de modelos, tanto en sus estructuras fijas como aleatorias, se realizó siguiendo el protocolo propuesto por Zuur et al. (2009). Como marco inferencial, se utilizó la teoría de información y se aplicó el criterio de Akaike (1973) (AIC) para la selección del mejor modelo (Burnham y Anderson 2002). Una vez obtenidos los modelos finales, se analizaron los puntos atípicos de los mismos y se eliminaron aquellos que fueran significativos.

Se evaluó la significancia de los factores fijos y se calculó el grado de bondad de ajuste y las varianzas explicadas por las componentes fija y aleatoria de los modelos mediante el coeficiente de determinación marginal, $\mathrm{R}^{2} \mathrm{~m}$, que representa la varianza explicada por la componente fija y el condicional, $\mathrm{R}^{2} \mathrm{c}$, que se interpreta como la varianza explicada por ambas componentes, la fija y la aleatoria (Nakagawa y Schielzeth 2013).
Se realizó un análisis de residuales sobre el modelo completo (Bolker et al. 2009; Zuur et al. 2009), a fin de validar los supuestos de los MLM. Con el fin de evaluar la variabilidad de los efectos aleatorios, se identificaron y aislaron las combinaciones lineales con menor sesgo (BLUP) y se estimó el valor del intercepto para cada nivel de los efectos aleatorios (Bates y Maechler 2009; Bates 2010).

Por último, se procedió a la extracción y análisis de los valores medios de la CPUE estandarizados y límites de sus respectivos intervalos de confianza correspondientes al factor AÑO. A fines comparativos, se analizaron y compararon los valores de CPUE estimados de cada modelo y a su vez, se contrastó con el modelo utilizado hasta la actualidad estimado por Zavatteri y Giussi (2019), este último realizado a partir de información extraída de la estadística pesquera. Para ello, se calculó el coeficiente de correlación de Pearson entre valores de CPUE estimados a partir de los diferentes modelos. Para comparar las tendencias generales, se ajustaron regresiones lineales a las CPUE estandarizadas por cada modelo y se compararon las pendientes a través del cociente de verosimilitud. Además, para comparar la tendencia año a año, se graficaron los residuales de cada regresión.

Todos los análisis fueron llevados a cabo utilizando la plataforma de programación $\mathrm{R}$ versión 3.6.1 (R Core Team 2019). Las pruebas fueron de dos colas con un nivel de significancia de $\mathrm{P} \leq 0,05$.

\section{RESULTADOS}

\section{Estimación y diagnóstico de modelos}

Se incluyeron 11.687 datos provenientes de observadores a bordo en el modelo lineal mixto $\left(\mathrm{MLM}_{\mathrm{OBS}}\right)$. Estos datos pertenecieron a seis buques surimeros que operaron sobre 17 áreas (Figura 1 A). Fueron excluidas 21 áreas que no cumplieron con los criterios de selección. Del 

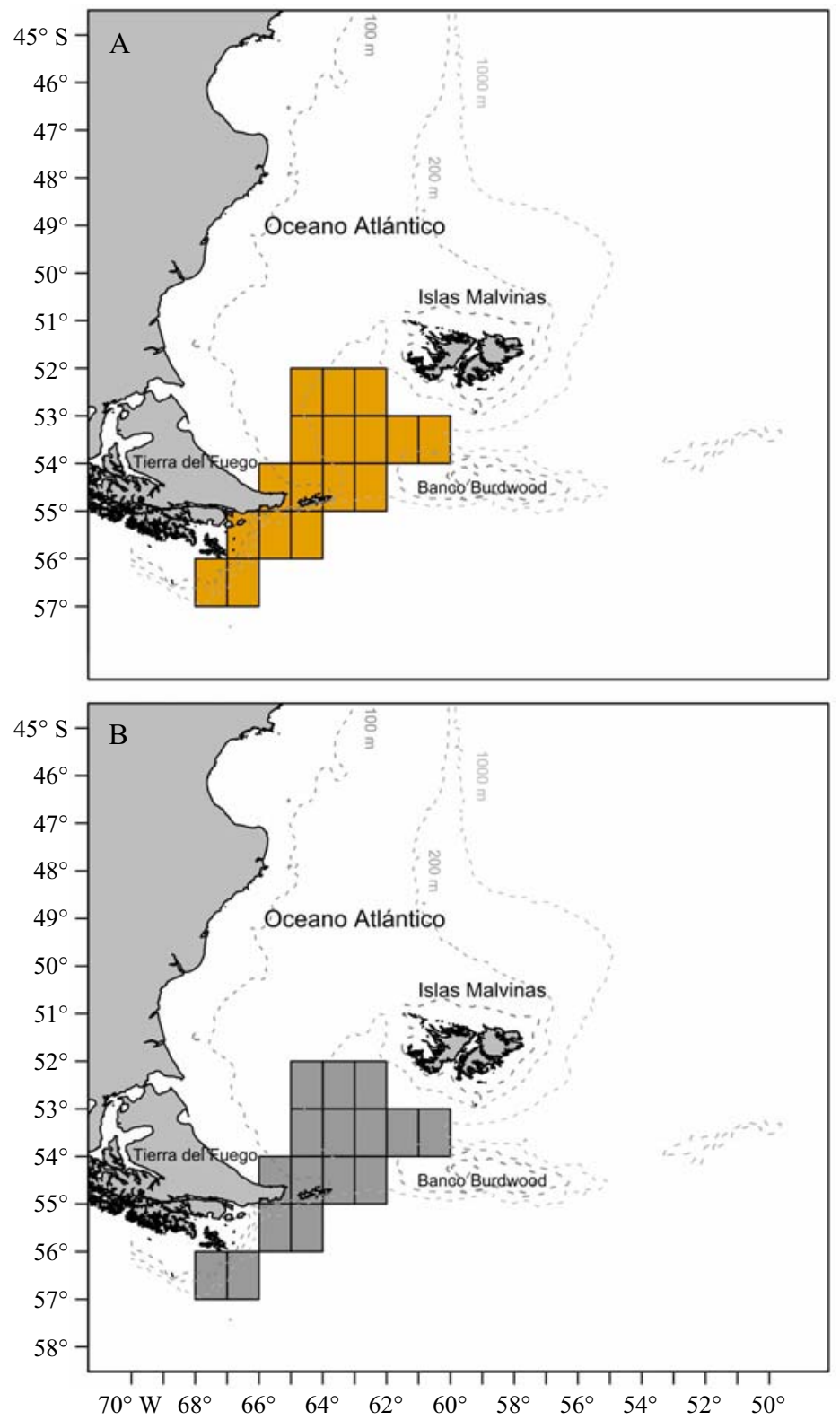

Figura 1. Mapa que indica las áreas (rectángulos estadísticos) incluidas en los modelos lineales mixtos (MLM) a partir de la información proveniente de observadores a bordo (A) y la información proveniente de la estadística pesquera (B).

Figure 1. Areas (statistical rectangles) included in the linear mixed models (LMM) from information from observers on board (A), and information from fishery statistics (B).

total de registros de la estadística oficial, 2.044 resultaron aptos para su posterior análisis e inclusión en el MLM (MLM $\mathrm{EST}_{\mathrm{T}}$, correspondientes a seis buques surimeros, que operaron sobre
16 áreas (Figura 1 B). En este caso se excluyeron 15 áreas que no cumplieron con la cantidad de registros.

Respecto de la selección de la componente 
aleatoria óptima, a partir de la comparación de modelos con distinta componente aleatoria y considerando la misma componente fija, el modelo más complejo que incluyó el factor aleatorio BUQUE y la interacción aleatoria AÑ $\times$ ÁREA resultó ser el mejor con ambas fuentes de información (Tabla 1). Al realizar la selección de la estructura en la componente fija, el modelo que incluyó todas las variables tuvo un peso relativo de aproximadamente el $100 \%$, por lo que fue elegido como el que mejor explicó la variabilidad de la CPUE. Esto fue observado para ambos modelos considerados (Tabla 2).

Los modelos ajustados quedaron definidos de la siguiente manera:

$\ln \left(\mathrm{CPUE}_{\mathrm{ijkl}}\right)=$ Intercepto $+\mathrm{ANO}_{\mathrm{i}}+\mathrm{MES}_{\mathrm{j}}+$ $\mathrm{ÁREA}_{\mathrm{k}}+\left(\mathrm{BUQUE}_{\mathrm{l}}\right)+\left(\mathrm{AÑO}_{\mathrm{i}} \times \hat{A R E A}_{\mathrm{k}}\right)+\varepsilon_{\mathrm{ijkl}}$

siendo:

$\varepsilon_{\mathrm{ijkl}} \sim \mathrm{N}\left(0, \sigma_{\varepsilon}\right)$

$\left(\mathrm{BUQUE}_{1}\right) \sim \mathrm{N}\left(0, \sigma_{\mathrm{BUQUEl}}\right)$;

$\left(\mathrm{ANO}_{\mathrm{i}} \times \mathrm{ÁREA}_{\mathrm{k}}\right) \sim \mathrm{N}\left(0, \sigma_{\mathrm{ANNO} \text { i } \times \text { ÁREAk }}\right)$.
El grado de bondad de ajuste de ambos modelos fue bueno $\left(\mathrm{MLM}_{\mathrm{OBS}}: \mathrm{R}_{\mathrm{m}}{ }^{2}=0,27, \mathrm{R}_{\mathrm{c}}{ }_{\mathrm{c}}=0,46\right.$; MLM $\left._{\mathrm{EST}}: \mathrm{R}_{\mathrm{m}}{ }^{2}=0,26, \mathrm{R}_{\mathrm{c}}{ }^{2}=0,38\right)$. La varianza explicada por la componente aleatoria en el MLM $_{\mathrm{OBS}}$ fue del $41 \%$ y del $31 \%$ en el MLM $\mathrm{EST}_{\text {. }}$. Todos los factores incluidos en ambos modelos re-sultaron estadísticamente significativos $\left(\mathrm{MLM}_{\mathrm{OBS}}: \mathrm{F}_{\mathrm{AÑO}}=6,63, \mathrm{gl}=25, \mathrm{p}<0,001, \mathrm{~F}_{\mathrm{MES}}\right.$ $=65,51, \mathrm{gl}=11, \mathrm{p}<0,001 ; \mathrm{F}_{\text {ÁREA }}=18,37, \mathrm{gl}=$ $16, \mathrm{p}<0,001 ; \mathrm{MLM}_{\mathrm{EST}}: \mathrm{F}_{\mathrm{AN} O}=9,60, \mathrm{gl}=25, \mathrm{p}$ $<0,001, \mathrm{~F}_{\mathrm{MES}}=23,92, \mathrm{gl}=11, \mathrm{p}<0,001 ; \mathrm{F}_{\text {ÁREA }}$ $=9,33, \mathrm{gl}=15, \mathrm{p}<0,001)$.

No se observó tendencia o patrón en los errores de los modelos, lo que indicó que se cumpliría de forma aceptable el supuesto de homocedasticidad (Figura 2). La gráfica de la probabilidad normal de los residuos evidenció una cierta aproximación a la distribución normal. En ambos modelos, la mayoría de los residuos se encontraron sobre la línea teórica de normalidad, excepto en el extremo inferior izquierdo donde los puntos se alejaron del intervalo de confianza (Figura 2).

Al analizar los efectos aleatorios en ambos modelos, se observó que para el factor BUQUE

Tabla 1. Número de parámetros $\left(\mathrm{k}_{\mathrm{p}}\right)$, delta de $\mathrm{AIC}(\Delta \mathrm{AIC})$ y peso relativo $\left(\omega_{\mathrm{i}}\right)$ de los modelos aplicados a la polaca, con diferentes estructuras aleatorias. En todos los modelos, los factores fijos fueron AÑO, MES y ÁREA. El AIC del mejor modelo (en negrita) del $\mathrm{MLM}_{\mathrm{OBS}}$ fue 45.361,5 y el del $\mathrm{MLM}_{\mathrm{EST}}$ 6.318,3. El signo “_” representa la modelo sin variables aleatorias (ML).

Table 1. Number of parameters $\left(k_{p}\right)$, AIC delta $(\triangle A I C)$ and relative weight $\left(\omega_{i}\right)$ of models applied to the Southern blue whiting with different random structures. Fixed factors were YEAR, MONTH and AREA in all models. AIC of the best model (bold) of MLM $M_{O B S}$ was 45,361.5 and that of MLM $M_{E S T}$ was 6,318.3. Sign "-" represents the model without random variables $(M L)$.

\begin{tabular}{llrrr}
\hline Modelo & \multicolumn{1}{c}{ Factor aleatorio } & $\mathrm{k}_{\mathrm{p}}$ & \multicolumn{1}{c}{$\Delta$ AIC } & $\omega_{\mathrm{i}}$ \\
\hline MLM $_{\mathrm{OBS}}$ & BUQUE + ÃNO $\times$ ÁREA & $\mathbf{5 6}$ & $\mathbf{0 , 0 0}$ & $\mathbf{1 , 0 0 0}$ \\
& AÑO ÁREA & 55 & 717,02 & 0,000 \\
& BUQUE & 55 & 949,21 & 0,000 \\
MLM $_{\mathrm{EST}}$ & - & 54 & $1.550,22$ & 0,000 \\
& BUQUE + Ã̃ O $\times$ ÁREA & $\mathbf{5 5}$ & $\mathbf{0 , 0 0}$ & $\mathbf{0 , 9 9 6}$ \\
& BUQUE & 54 & 10,86 & 0,004 \\
& - & 53 & 52,17 & 0,000 \\
& AÑO $\times$ ÁREA & 54 & 182,52 & 0,000 \\
\hline
\end{tabular}


Tabla 2. Número de parámetros $\left(\mathrm{k}_{\mathrm{p}}\right)$, delta de $\mathrm{AIC}(\triangle \mathrm{AIC})$ y peso relativo $\left(\omega_{\mathrm{i}}\right)$ de los distintos modelos, aplicados a la polaca. En todos los modelos, las variables aleatorias fueron BUQUE y la interacción AÑO $\times$ ÁREA. El AIC del mejor modelo (en negrita) del $\mathrm{MLM}_{\mathrm{OBS}}$ fue 45.275,5 y el del MLM $\mathrm{EST}_{6.192,8 .}$

Table 2. Number of parameters $(\mathrm{kp})$, AIC delta $(\triangle A I C)$ and relative weight $\left(\omega_{i}\right)$ of different models applied to the Southern blue whiting. Random variables were VESSEL and the YEAR $\times$ AREA interaction in all models. AIC of the best model (bold) of $M L M_{O B S}$ was 45,275.5 and that of $M L M_{E S T}$ was 6,192.8.

\begin{tabular}{|c|c|c|c|c|}
\hline Modelos & Factores fijos & $\mathrm{k}_{\mathrm{p}}$ & $\triangle \mathrm{AIC}$ & $\omega_{\mathrm{i}}$ \\
\hline \multirow[t]{8}{*}{ MLM $_{\mathrm{OBS}}$} & AÑNO + MES + ÁREA & 56 & $\mathbf{0 , 0 0}$ & 1 \\
\hline & $\mathrm{MES}+$ ÁREA & 31 & 101,09 & 0 \\
\hline & AÑO + MES & 40 & 196,72 & 0 \\
\hline & MES & 15 & 237,72 & 0 \\
\hline & AÑO + ÁREA & 45 & 686,44 & 0 \\
\hline & ÁREA & 20 & 788,98 & 0 \\
\hline & AÑO & 29 & 836,58 & 0 \\
\hline & NULO & 4 & 887,83 & 0 \\
\hline \multirow[t]{8}{*}{$\mathrm{MLM}_{\mathrm{EST}}$} & AÑNO + MES + ÁREA & 55 & $\mathbf{0 , 0 0}$ & 1 \\
\hline & AÑO + MES & 40 & 118,43 & 0 \\
\hline & MES + ÁREA & 30 & 173,96 & 0 \\
\hline & MES & 44 & 221,44 & 0 \\
\hline & AÑO + ÁREA & 15 & 221,70 & 0 \\
\hline & AÑO & 29 & 331,43 & 0 \\
\hline & ÁREA & 19 & 375,27 & 0 \\
\hline & NULO & 4 & 421,61 & 0 \\
\hline
\end{tabular}

se encontraron predicciones de los niveles y sus intervalos de confianza ubicados en lados opuestos de la línea del intercepto fijo, lo que demuestra variabilidad entre niveles. Para el caso de la interacción AÑO $\times$ ÁREA en el $\mathrm{MLM}_{\mathrm{OBS}}$, se encontraron niveles e intervalos de confianza ubicados en lados opuestos del intercepto fijo, en cambio, en el MLM $\mathrm{EST}_{\mathrm{T}}$ las predicciones se encontraron en niveles opuestos del intercepto fijo, pero no así sus intervalos de confianza, los cuales lo incluyeron en su totalidad evidenciando poca variabilidad (Figura 3).

\section{Análisis de tendencias}

En general, el comportamiento de los valores medios anuales de la CPUE estandarizada extraí- dos a partir del MLM $_{\mathrm{OBS}}$ evidenció dos períodos, uno correspondiente a los años 1993-2004 con valores por encima de la media (1.212,39 $\left.\mathrm{kg} \mathrm{h}^{-1}\right)$, y otro a los años 2005-2018 con valores por debajo de la misma (Figura $4 \mathrm{~A}$ ). Los valores medios estandarizados de la CPUE por año a partir del $\mathrm{MLM}_{\mathrm{EST}}$ evidenciaron tres períodos comprendidos entre los años 1993-2004, 2005-2014 y 2015 2018 (Figura 4 B). En el primer y último período, los valores medios de CPUE fueron mayores a la media general $\left(2.322,4 \mathrm{~kg} \mathrm{~h}^{-1}\right)$ mientras que en el segundo, estos valores fueron menores.

\section{Comparación de tendencias}

El coeficiente de correlación de Pearson entre las CPUE estimadas a partir del MLM $_{\mathrm{EST}} \mathrm{y}$ el 


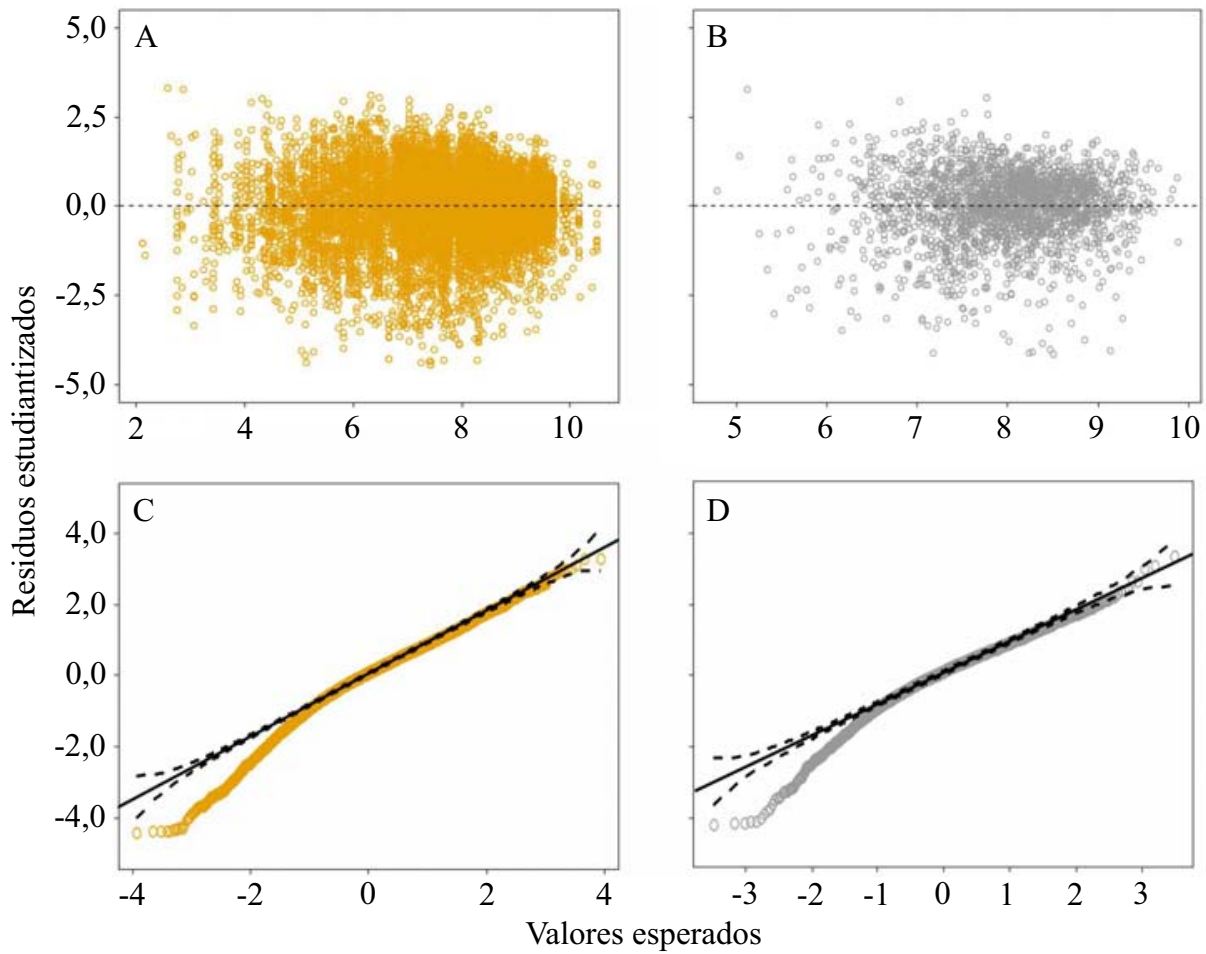

Figura 2. Distribución de los residuos estandarizados por sus desviaciones estándares respecto de los valores estimados a partir

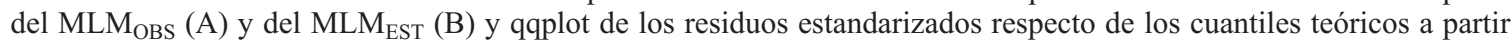
del $\mathrm{MLM}_{\mathrm{OBS}}$ (C) y del MLM $\mathrm{EST}$ (D). La línea recta continua indica la probabilidad teórica normal y la línea punteada sus intervalos de confianza del $95 \%$.

Figure 2. Distribution of standardized residuals by their standard deviations with respect to the values estimated from $M L M_{O B S}$ (A) and $M L M_{E S T}(B)$ and qqplot of standardized residuals with respect to the theoretical quantiles from $M L M_{O B S}(C)$ and $M L M_{E S T}(D)$. Solid straight line indicates the normal theoretical probability and the dotted line its $95 \%$ confidence intervals.

$\mathrm{MLM}_{\mathrm{OBS}}$ fue de 0,62 y significativamente diferente de cero $\left(t_{24}=3,93, p<0,01\right)$, lo que demostró una correlación positiva entre las mismas (Figura 5 A). Por otro lado, las correlaciones entre las CPUE medias estimadas a partir de los modelos construidos en este trabajo y las obtenidas a partir del modelo propuesto por Zavatteri y Giussi (2019), fueron positivas y significativamente diferentes de cero $\left(r_{\mathrm{MLMobs}}=0,56, \mathrm{t}_{24}=\right.$ $3,35, \mathrm{p}<0,01 ; r_{\text {MLMest }}=0,92, \mathrm{t}_{24}=12,08, \mathrm{p}<$ 0,001 ; Figura 5 B y 5 C). No hubo diferencias significativas entre las pendientes estimadas en las tres estimaciones $\left(x^{2}{ }_{1}=1,08, \mathrm{p}=0,58\right.$; Figura 6 A) y la tendencia entre las tres estimaciones fue relativamente similar, excepto en 1993 y 1998 en donde la tendencia de la CPUE estimada por el $\mathrm{MLM}_{\mathrm{OBS}}$ fue contraria a las otras (Figura $6 \mathrm{~B}$ ).

\section{DISCUSIÓN}

En este trabajo se estandarizaron las capturas por unidad de esfuerzo de la polaca provenientes de dos fuentes de información empleando modelos lineales mixtos. Además, los índices así obtenidos fueron comparados entre sí y con el modelo que se desarrolla anualmente para estimar índices de abundancia de polaca en el INIDEP (Giussi y Zavatteri 2017; Zavatteri y Giussi 2018, 2019). Si 

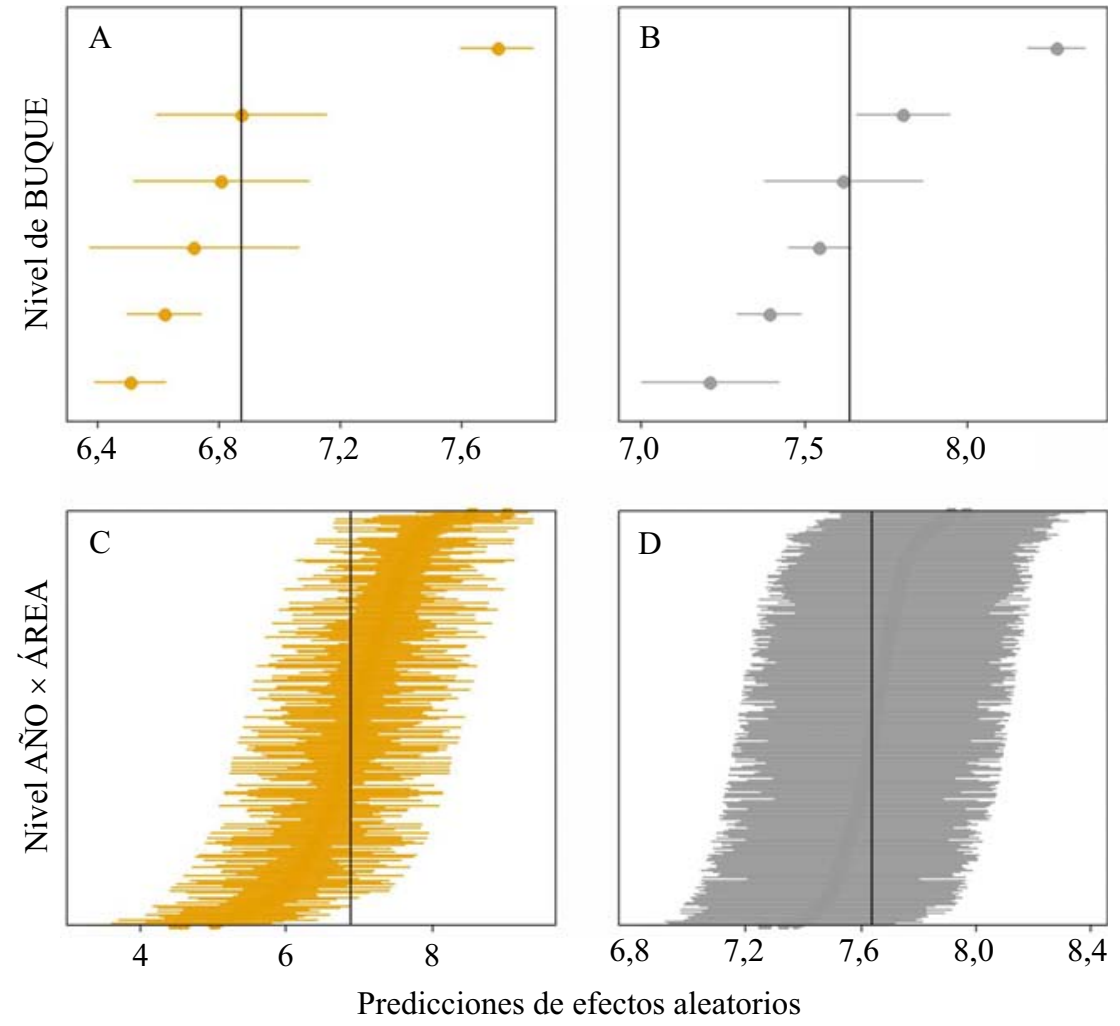

Figura 3. Gráfico de "oruga" de las predicciones de intercepto de los efectos aleatorios BUQUE a partir del MLM OBS $_{\text {(A) y del }}$ $\mathrm{MLM}_{\mathrm{EST}}(\mathrm{B})$ e interacción AÑO $\times$ ÁREA a partir del $\mathrm{MLM}_{\mathrm{OBS}}(\mathrm{C})$ y del $\mathrm{MLM}_{\mathrm{EST}}$ (D) ajustados a polaca y sus intervalos de confianza del $95 \%$. La línea vertical representa el intercepto fijo para cada modelo.

Figure 3. "Caterpillar" graph of the intercept predictions of the random effects VESSEL from MLM $O B S(A)$ and MLM $M_{E S T}(B)$ and $Y E A R \times A R E A$ interaction from $M L M_{O B S}(C)$ and $M L M_{E S T}(D)$ adjusted to the Southern blue whiting and their $95 \%$ confidence intervals. Vertical line represents the fixed intercept for each model.

bien dicha comparación había sido realizada por Giussi et al. (2012), la construcción del índice de abundancia con MLM no había sido reportada para la especie en la Argentina hasta la actualidad. Estos tipos de modelos permiten mejorar la calidad del análisis, ya que pueden modelar la variabilidad aleatoria y la correlación de los errores. Esto resulta muy útil en el análisis de datos desbalanceados con pseudoréplicas o con algún tipo de estructura jerárquica o de agrupación, los cuales suelen estar presentes al elaborar índices de abundancia (Di Marco et al. 2019). Los modelos construidos con ambas fuentes de información que mejor explicaron la variabilidad de la CPUE incluyeron las mismas variables tanto en su estructura fija como aleatoria. Ambos tuvieron un buen ajuste y la variación explicada fue mayor respecto de los índices de abundancia anteriores (Giussi y Zavatteri 2017; Zavatteri y Giussi 2018, 2019).

En el $\mathrm{MLM}_{\mathrm{OBS}}$, se observó una alta variación de la CPUE tanto entre buques como entre las interacciones AÑ $\times$ ÁREA, por lo que se justificó la inclusión de dichas variables como aleatorias, mientras que, en el MLM $\mathrm{EST}_{\mathrm{E}}$ se observó variación entre buques, pero no así entre las interacciones AÑO $\times$ ÁREA. Esto mismo se vio reflejado en la varianza explicada por la variable aleatoria, siendo un $41 \%$ del total en el MLM OBS $_{\text {y }}$ un $31 \%$ del total

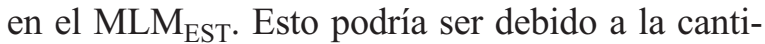
dad de registros provenientes de ambas fuentes de 


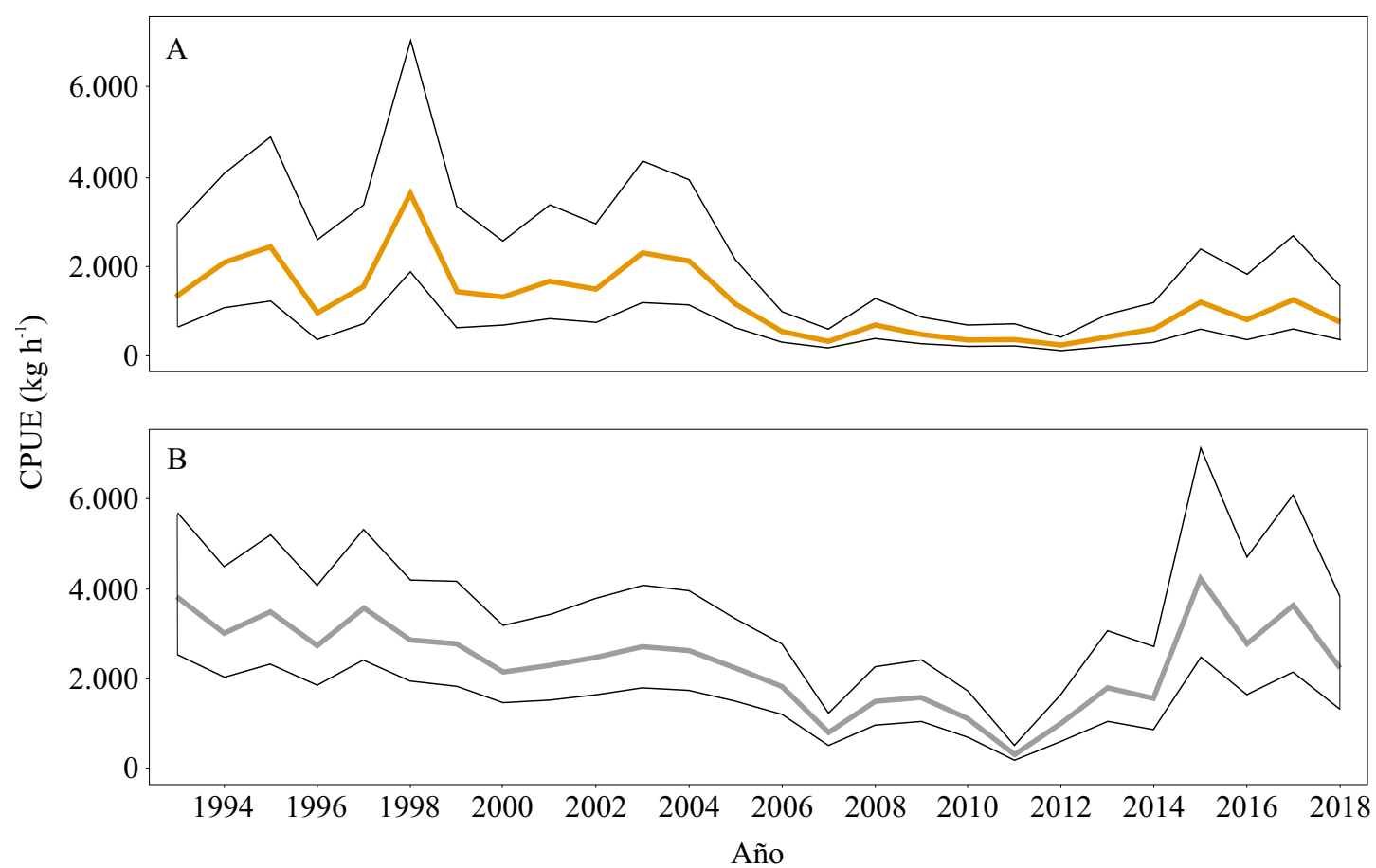

Figura 4. Valores medios e intervalos de confianza del 95\% de la captura por unidad de esfuerzo (CPUE) estandarizada, corres-

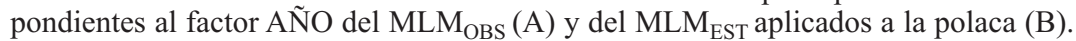

Figure 4. Mean values and 95\% confidence intervals of the standardized catch per unit effort (CPUE) corresponding to YEAR factor of $M L M_{O B S}(\mathrm{~A})$ and $M L M_{E S T}$ applied to the Southern blue whiting (B).
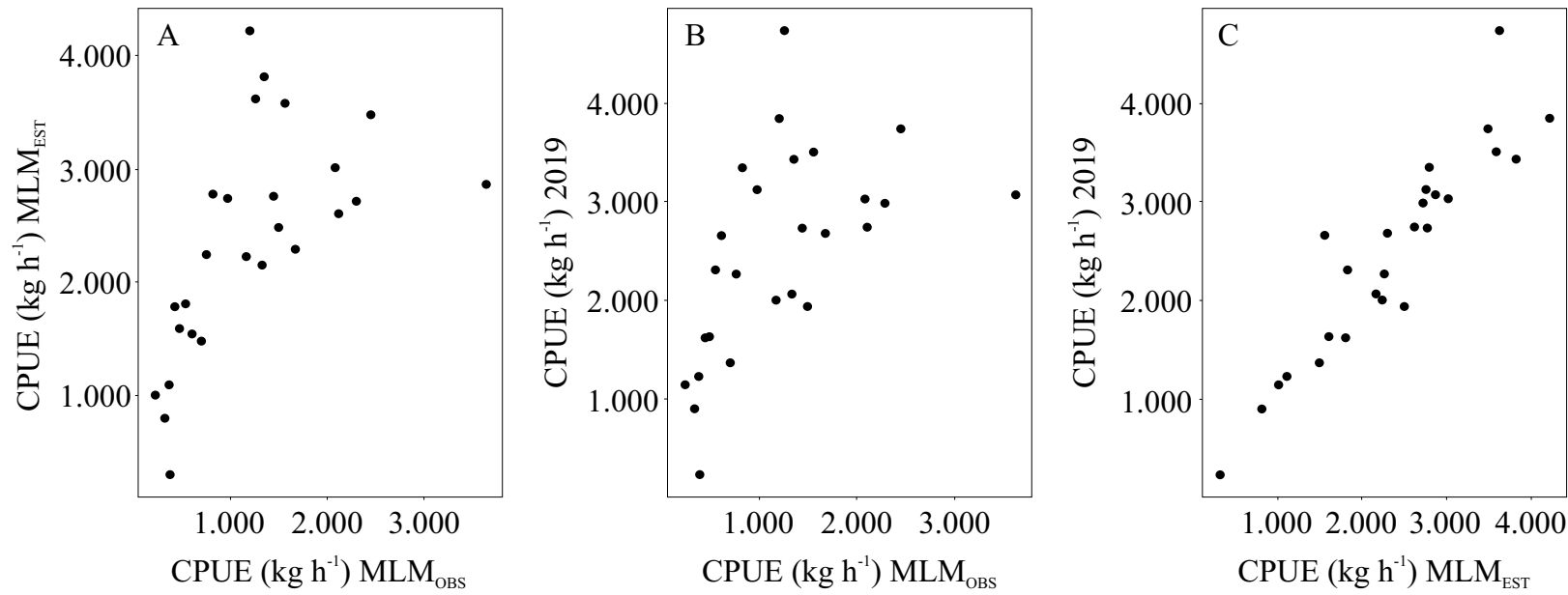

Figura 5. Diagrama de dispersión entre las tendencias de la captura por unidad de esfuerzo (CPUE) estimadas a partir del MLM $_{\mathrm{OBS}}$ versus el MLM $\mathrm{EST}_{\text {(A), el MLM }}$ OBS versus el ML estimado por Zavatteri y Giussi (2019) (B) y el MLM versus el ML estimado por Zavatteri y Giussi (2019) (C).

Figure 5. Scatter diagram between the estimated catch per unit effort (CPUE) trends from MLM $M_{O B S}$ versus $M L M_{E S T}(A)$, MLM $O B S$ versus ML estimated by Zavatteri and Giussi (2019) (B), and MLMEST versus estimated ML by Zavatteri and Giussi (2019) (C). 

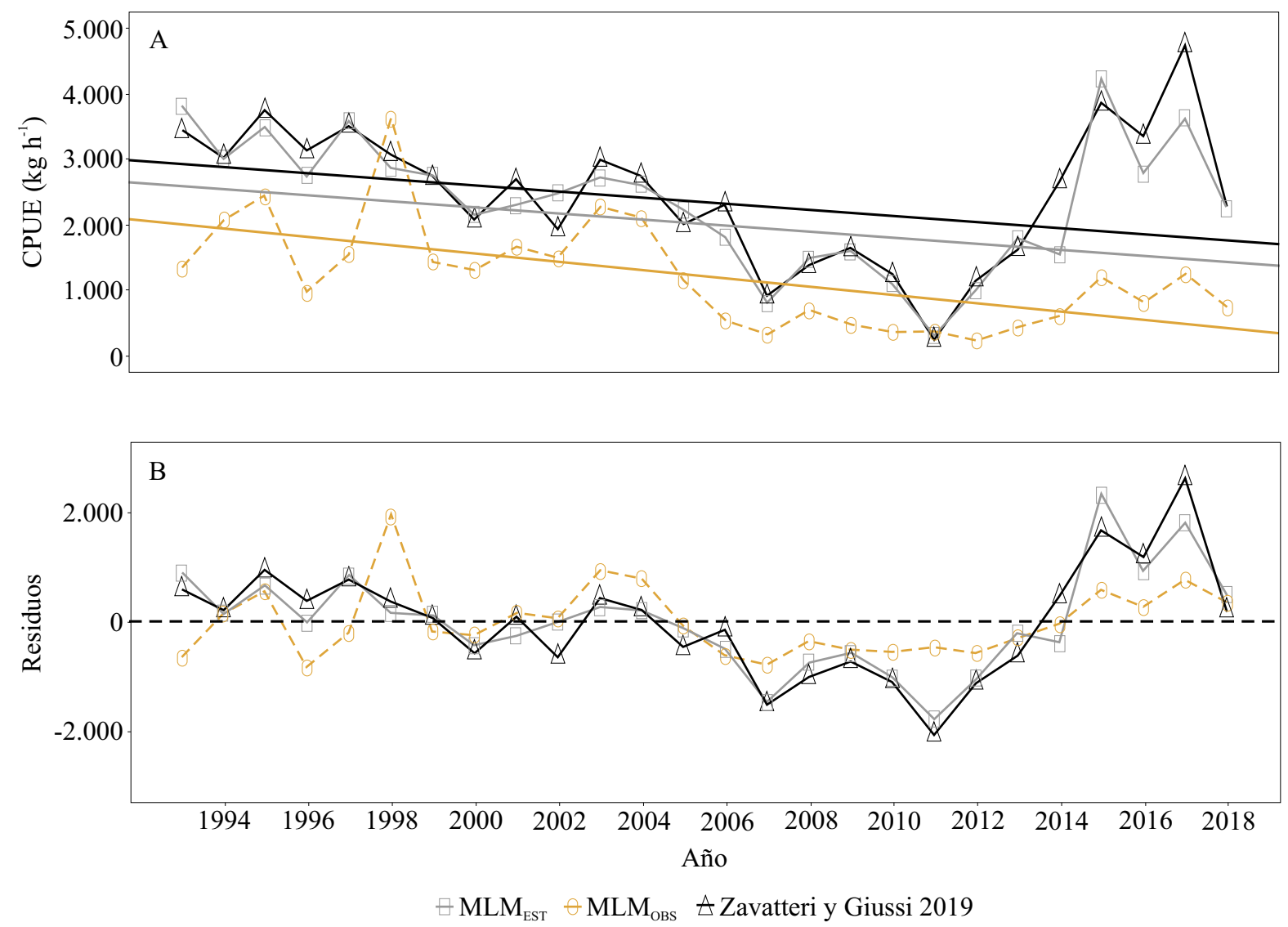

Figura 6. Comparación de tendencias de las capturas por unidad de esfuerzo (CPUE) estimadas a partir del MLM $_{\mathrm{OBS}}$, el MLM $\mathrm{EST}_{\mathrm{B}}$ y el ML estimado por Zavatteri y Giussi (2019). A) CPUE estandarizadas y sus respectivas líneas de tendencias estimadas. B) Comparación de los residuales para cada tendencia, la línea punteada horizontal representa la media de los residuos.

Figure 6. Comparison of trends in the estimated catch per unit effort (CPUE) from MLM OBS, MLM EST and ML estimated by Zavatteri and Giussi (2019). A) Standardized CPUE and their respective estimated trend lines. B) Comparison of the residuals for each trend, horizontal dotted line represents the mean of the residuals.

información, las cuales son mayores en los datos tomados por los observadores a bordo de buques comerciales que las obtenidas por los partes de pesca, lo que conduciría a una estimación más representativa de la CPUE, explicando así una mayor variabilidad de los datos observados.

En cuanto a los valores medios de CPUE estandarizado por año a partir del $\mathrm{MLM}_{\mathrm{OBS}}$, se registró una alta variabilidad entre 1993 y 2005, año a partir del cual la tendencia se mostró más estable. En el caso de los valores medios por año estimados por el $\mathrm{MLM}_{\mathrm{EST}}$ en contraposición con los estimados por el MLM $\mathrm{OBBS}_{\mathrm{OBS}}$, se observó una variabilidad menor durante el primer período comprendido entre 1993 y 2006. Sin embargo, a pesar de las diferencias interanuales en los valores medios de CPUE estimados con ambos modelos, la tendencia general resultó similar. De igual forma, las CPUE medias estimadas a partir de los modelos lineales mixtos planteados utilizando ambas fuentes de información tuvieron una alta correlación con los valores obtenidos por Zavatteri y Giussi (2019) a partir de un modelo lineal. Esto indicaría que el enfoque mediante modelos 
mixtos no generaría un cambio sobre la tendencia de los índices de abundancia estimados a través de modelos lineales simples.

Por otro lado, teniendo en cuenta que para la realización del MLM $_{\mathrm{EST}} \mathrm{y}$ el ML estimado por Zavatteri y Giussi (2019) se utilizó la misma base de datos, la relación entre las CPUE así estimadas resultaría de gran utilidad a modo comparativo. La tendencia entre estos dos modelos fue muy similar, con un alto nivel de correlación, aunque la tendencia estimada por el MLM EST $_{\text {fue menos varia- }}$ ble. Además, el ajuste del MLM $_{\mathrm{EST}}$ fue mejor y utilizó una menor cantidad de parámetros, contribuyendo así a una mayor parsimonia del modelo.

Debido a lo anterior, se podría concluir que la aplicación de MLM mejoró el ajuste de los modelos lineales utilizados hasta el momento sin variar abruptamente la tendencia estimada, lo cual permitiría predecir con mayor exactitud los valores medios de CPUE estandarizados a lo largo de los

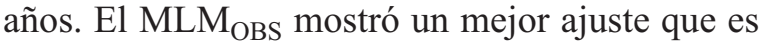
explicado por el mayor número de registros y mayor variabilidad de los datos respecto del

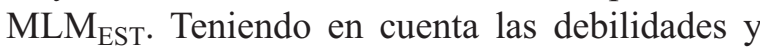
fortalezas en cuanto a la representatividad de las dos fuentes de información consideradas, se recomienda utilizar ambas para lograr estimaciones más certeras y mejorar la calibración de los modelos de evaluación empleados en la estimación de abundancia de la especie.

\section{AGRADECIMIENTOS}

Los autores agradecen a la Dra. Analía Giussi por la lectura y aportes realizados al manuscrito. A los integrantes del "Programa de Pesquerías de Peces Demersales, Australes y Subantárticos", por la colaboración. También queremos agradecer a los tres revisores anónimos, quienes con sus comentarios y sugerencias ayudaron a mejorar la calidad de este trabajo. Contribución INIDEP $\mathrm{N}^{\circ}$ 2217.

\section{REFERENCIAS}

Aguayo M, Chong J, Payá I. 2010. Edad, crecimiento y mortalidad natural de merluza de tres aletas, Micromesistius australis en el Océano Pacífico suroriental. Rev Biol Mar Oceanogr. 45: 723-735.

AKAIKE H. 1973. Information theory and an extension of the Maximum Likelihood Principle. En: Petrov, BN, Csaki F, editores. Second International Symposium on Information Theory. p. 267-281.

BATES D. 2010. lme4: mixed-effects modeling with R. http://lme4.r-forge.r-project.org/book.

Bates D, Maechler M. 2009. Ime4 Linear Mixed-Effects Models Using S4 Classes. R package version 0.999375-32.

Bolker B, Brooks M, Clark C, Geange S, Poulsen J, Stevens HS, White J. 2009. Generalized Linear Mixed Models: a practical guide for ecology and evolution. Trends Ecol Evol. 24 (3): 127-135.

BURNHAM KP, ANDERSON DR. 2002. Model selection and multimodel inference: a practical information-theoretic approach. 2da ed. Nueva York: Springer. 488 p.

Céspedes R, Adasme L, Gálvez P, Boré D, Tascheri R, Montenegro C, Bravo C, Roвотнам H, Zuleta A. 1998. Análisis de la pesquería de merluza de tres aletas en la zona sur-austral. Informe Final Proyecto FIP 199639. Fondo de Investigación Pesquera, Valparaíso, Chile.

Chiechomsky JD, ERlich MD, LAsta CA, SÁNCHEZ RP. 1981. Distribución de huevos y larvas de peces en el Mar Epicontinental Argentino y evaluación de los efectivos desovantes de anchoíta y merluza. Contrib Inst Nac Invest Desarr Pesq (Mar del Plata). N ${ }^{\circ}$ 383: 59-79.

Cohen D, Inada T, Iwamoto T, Scialabba N. 1990. Gadiform fishes of the world (Order 
Gadiformes). FAO Species Catalogue. FAO Fish Synop. 125 (10): 1-442.

CORdo HD, WöHLER OC. 1998. Estimación de índices de abundancia de la polaca (Micromesistius australis) a partir de información proveniente de la flota comercial argentina. Inf Téc INIDEP N 119/1998. 14 p.

Cousseau MB. 1993. Las especies del Orden Gadiformes del Atlántico Sudamericano comprendido entre $34^{\circ}$ y $55^{\circ} \mathrm{S}$ y su relación con las de otras áreas. Frent Marít. 13 (A): 7-108.

Crawley MJ. 2002. Statistical computing: an introduction to data analysis using S-Plus. Chichester: John Wiley and Sons Ltd. 772 p.

Di Marco E, Troccoli G, Martínez PA. 2019. Estandarización de la captura por unidad de esfuerzo de merluza negra (Dissostichus eleginoides) derivada de la flota arrastrera argentina mediante la aplicación de un modelo lineal mixto. Período 2010-2017. Inf Invest INIDEP $\mathrm{N}^{\circ} 65 / 2019.19 \mathrm{p}$.

Giussi AR, Di MARco E, WöHLER O, ZaVATTERI A. 2012. Validation of statistics cpue index of southern blue whiting (Micromesistius australis) by scientific observer data. 6th World Fisheries Congress "Sustainable Fisheries in a Changing World" (Sexto Congreso Mundial de Pesquerías); 7 y 11 de mayo de 2012; Edimburgo, Escocia. Pesentación en formato póster electrónico.

Giussi AR, Di MARco E, Zavatteri A, WÖHLER O. 2013. Estimación del índice de abundancia de polaca (Micromesistius australis) a partir de la captura por unidad de esfuerzo de buques surimeros argentinos. Período 1992-2012. Inf Téc INIDEP No 24/2013. 11 p.

Giussi AR, Zavatteri A. 2017. Estimación del índice de abundancia de polaca (Micromesistius australis) a partir de la captura por unidad de esfuerzo de buques surimeros argentinos. Período 1992-2016. Inf Téc INIDEP $\mathrm{N}^{\circ}$ 27/2017. 12 p.

Giussi AR, ZaVatteri A, Di Marco E. 2016. Estimación del índice de abundancia de polaca
(Micromesistius australis) a partir de la captura por unidad de esfuerzo de buques surimeros argentinos. Período 1992-2014. Inf Téc INIDEP N ${ }^{\circ} 3 / 2016.10 \mathrm{p}$.

Gorini FL, GiUSSI AR. 2018. Estadística pesquera de peces demersales australes en el Atlántico Sudoccidental (Período 2005-2017). Inf Téc INIDEP No 25/2018. 62 p.

Gorini FL, GiUssi AR. 2019. Estadística pesquera de peces demersales australes en el Atlántico Sudoccidental (Período 2006-2018). Inf Téc INIDEP N ${ }^{\circ} 33 / 2019.62$ p.

HERNÁNDEZ DR. 2004. Estimación de índices de abundancia relativa, estimación del poder de pesca y estandarización del esfuerzo a partir de modelos multiplicativos. Notas de Divulgación Año 2004. Revisión y ampliación de notas del 2002. Biblioteca INIDEP. 55 p.

Kock KH, Duhamel G, Hureau JC. 1985. The biology and present status of exploited Antarctic fish stocks: a review. Biomass Sci Ser. 6: 1143.

Lillo S, CÉSPEDEs R, BARBIERI M. 1999. Evaluación directa del stock de merluza de tres aletas (Micromesistius australis) y monitoreo de sus procesos biológicos y migratorios. Informe Final, IFOP. 48 p.

Lillo S, Céspedes R, OJeda V, SaAvedra A. 2005. Evaluación hidroacústica del stock parental de merluza de tres aletas em su unidad de pesquería, año 2004 (Fase I). Informe Final, Proyecto FIP 2004-08-1: 1-104.

Nakagawa S, Schielzeth H. 2013. A general and simple method for obtaining $\mathrm{R}^{2}$ from Generalized Linear Mixed-effects Models. Methods Ecol Evol. 4: 133-142.

Perrotta, RG. 1982 Distribución y estrutura poblacional de la polaca (Micromesistius australis). Rev Invest Desarr Pesq. 3: 35-50.

Pinheiro JC, BATEs DM. 2000. Mixed-effectsmodels in S and S-PLUS. Nueva York: Springer. $528 \mathrm{p}$.

R Core Team. 2019. R: A language and environment for statistical computing. R Foundation 
for Statistical Computing, Vienna, Austria. https://www.r-project.org/.

WeIss G. 1974. Hallazgo y descripción de larvas de polaca (Micromesistius australis) en aguas del sector patagónico (Pisces, Gadidae). Physis A. 33 (87): 537-542.

WöHler OC, CAssia MC, Hansen JE. 2004. Caracterización biológica y estado de explotación de la polaca (Micromesistius australis). En: SÁnchez RP, Bezzi SI, editores. El Mar Argentino y sus recursos pesqueros. Tomo 4. Los peces marinos de interés pesquero. Caracterización biológica y evaluación del estado de explotación. Mar del Plata: Instituto Nacional de Investigación y Desarrollo Pesquero (INIDEP): 283-305.

Wöhler OC, Cordo HD, Hansen JE, Cassia MC. 2001. Análisis secuencia de la población de polaca (Micromesistius australis) en el período 1987-1997, ajustado con valores de rendimiento por unidad de esfuerzo de pesqueros comerciales. Rev Invest Desarr Pesq. 14: 37-56.
Wöhler OC, Hansen Je, Cassia MC, Cordo HD. 2007. Evaluación de polaca (Micromesistius australis) en el Atlántico Sudoccidental. Período 1987-2001. INIDEP Inf Téc. 62: $27-$ 52.

Zavatteri A, Giussi AR. 2018. Estimación del índice de abundancia de polaca (Micromesistius australis) a partir de la captura por unidad de esfuerzo de buques surimeros argentinos. Periodo 1992-2017. Inf Téc INIDEP $\mathrm{N}^{\circ}$ 42/2018. $11 \mathrm{p}$.

Zavatteri A, Giussi AR. 2019. Estimación del índice de abundancia de polaca (Micromesistius australis) a partir de la captura por unidad de esfuerzo de buques surimeros argentinos. Período 1992-2018. Inf Téc INIDEP $\mathrm{N}^{\mathrm{o}}$ 50/2019. 12 p.

ZuUR AF, Ieno EM, SMith GM. 2007. Analysing ecological data. Nueva York: Springer. 672 p. Zuur AF, Ieno EN, Walker N, Saveliev A, Smith G. 2009. Mixed Effect Models and extensions in ecology with R. doi:10.1007/ 978-0-387-87458-6 